\title{
An Algorithm for Fast Optimal Latin Hypercube Design of Experiments
}

\author{
Felipe A. C. Viana ${ }^{1}$ \\ University of Florida, Gainesville, FL 32611, USA \\ Gerhard Venter ${ }^{2}$ \\ Stellenbosch University, Matieland, Stellenbosch 7602, South Africa \\ and \\ Vladimir Balabanov ${ }^{3}$ \\ The Boeing Company, Seattle, WA 98204, USA
}

\begin{abstract}
This paper presents the Translational Propagation algorithm; a new method for obtaining optimal or near optimal Latin hypercube designs without using formal optimization. The procedure requires minimal computational effort with results virtually provided in real time. The algorithm exploits patterns of point locations for optimal Latin hypercube designs based on the $\phi_{p}$ criterion (a variation of the maximum distance criterion). Small building blocks, consisting of one or more points each, are used to recreate these patterns by simple translation in the hyperspace. Monte Carlo simulations were used to evaluate the performance of the new algorithm for different design configurations where both the dimensionality and the point density were studied. The proposed algorithm was also compared against three formal optimization approaches (namely random search, genetic algorithm, and enhanced stochastic evolutionary algorithm). It was found that (i) the distribution of the $\phi_{p}$ values tends to lower values as the dimensionality is increased; and (ii) the proposed translational propagation algorithm represents a computationally attractive strategy to obtain near optimum Latin hypercube designs up to medium dimensions.
\end{abstract}

Keywords: Design of Computer Experiments; Experimental design; Latin Hypercube Sampling; Translational Propagation Algorithm.

\footnotetext{
${ }^{1}$ (Corresponding author) Research Assistant, Dept. of Mechanical and Aerospace Engineering, fchegury@ufl.edu.

2 Professor, Dept. of Mechanical and Mechatronic Engineering, gventer@sun.ac.za.

${ }^{3}$ Structural Analysis Engineer, v.o.balabanov@gmail.com.
} 


\section{Introduction}

Design optimization usually requires a large number of potentially expensive simulations. Advancements in computational hardware and algorithms have not alleviated much of the resulting computational crunch because of the ever increasing appetite for improved modeling of physical processes and more detailed optimization [1]. To reduce the computational cost, surrogate models, also known as meta-models, are often used in place of the actual simulation models [2]-[7]. Surrogate-based design optimization begins by identifying locations in the design space where simulations will be conducted. This process of identifying locations in the design space is known as design of experiments (DOE) [8], [9]. Response data (often via numerical simulations) is collected at these locations and one or more candidate surrogate models are fitted to the data [10]-[12]. Finally, one or more of the candidate models are selected for calculating responses (and to facilitate objective and constraint calculation during the optimization process) at points in the design space where the actual responses are not yet available.

It is well known among designers that the quality of fit, which often defines the performance of the surrogate model during optimization and design space exploration strongly depends on the design of experiments (point location and density) [13], [14]. By quality of fit, the authors imply the discrepancy (in a general sense) between the actual response and the value predicted by the corresponding surrogate model. There exist many measures for the quality of fit, depending on the particular problem under consideration [14], [15].

A design of experiment with $n_{p}$ points and $n_{v}$ variables is usually written as a $n_{p} \times n_{v}$ matrix $\mathbf{X}=\left[\mathbf{x}_{1}, \mathbf{x}_{2}, \ldots, \mathbf{x}_{n_{p}}\right]^{T}$, where each row $\mathbf{x}_{i}=\left[x_{i 1}, x_{i 2}, \ldots, x_{i n_{v}}\right]$ represents a sample and each column represents a variable. Within the design and analysis of computer experiments, the Latin hypercube design (LHD) proposed by McKay et al. [16] and Iman and Conover [17], is very popular. The LHD presents advantages such as: (i) the number of samples (points) is not fixed; (ii) orthogonality of the sampling points (a design is orthogonal if the inner product of any two columns is zero [3], [18]); and (iii) the sampling points do not depend on the surrogate model that will be constructed. A LHD with $n_{p}$ points is constructed in such a way that each of the $n_{v}$ variables is divided into $n_{p}$ equal levels and that there is only one point (or sample) at each level. A random procedure is used to determine the point locations. Figure 1 shows two examples of LHDs with $n_{v}=2$ and $n_{p}=16$. Since the LHD is constructed using a random procedure, there is nothing preventing a design that has poor space filling qualities, as 
the extreme case illustrated in Figure 1-(a). A better choice is shown in Figure 1-(b), where the points are more uniformly distributed in the domain.

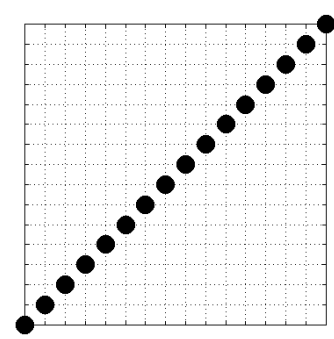

(a) Ill-suited LHD with $n_{v}=2$ and $n_{p}=16$.

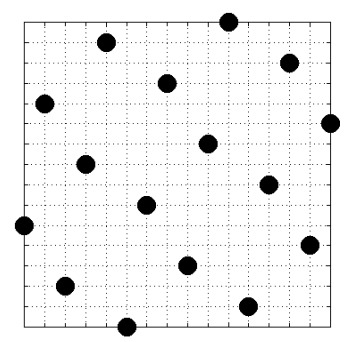

(b) Reasonable LHD with $n_{v}=2$ and $n_{p}=16$.

Figure 1: Examples of Latin hypercube designs.

The optimization of the space-filling qualities of the Latin hypercube design is a challenging problem that has resulted in a number of research publications [19]-[28]. One interpretation of the space filling property is to consider a $n_{v}$-dimensional sphere around each design point in the experimental design. The larger the radius of the smallest sphere that does not cross the boundary of the design space, the better the space filling property of the design. Optimizing the point location in a Latin hypercube design to improve the uniformity of the point distribution, typically by maximizing the radius of the smallest sphere, results in an Optimal Latin hypercube design. Such designs are usually obtained from time consuming combinatorial optimization problems, with search space of the order of $\left(n_{p} !\right)^{n_{v}}$. For example, to optimize the location of 20 samples in 2 dimensions, the algorithm has to select the best design from more than $10^{36}$ possible designs. If the number of variables is increased to 3 , the number of possible designs is more than $10^{55}$. Researches have proposed various optimization algorithms and objective functions to solve this problem. Table 1 summarizes some strategies found in the literature. As for the computational time, Ye et al. [22] reported several hours on a Sun SPARC 20 workstation for generating an optimal Latin hypercube with 25 points for 4 variables. Jin et al. [25] reported minutes on a PC with a Pentium III 650 MHZ CPU for generating an optimal Latin hypercube with 100 points for 10 variables. Section IV shows a comparison of different optimization methods for obtaining optimal LHDs with contemporary computing power.

Due to its popularity, the $\phi_{p}$ criterion was selected as a performance measure in this paper. Minimizing $\phi_{p}$ leads to the maximization of the point-to-point distance in the design (see [21] and [25] for details). Mathematically: 


$$
\phi_{p}=\left[\sum_{i=1}^{n_{p}-1} \sum_{j=i+1}^{n_{p}} d_{i j}^{-p}\right]^{1 / p},
$$

where $p$ is a positive integer, $n_{p}$ is the number of points in the design and $d_{i j}$ is the inter-point distance between all point pairs in the design. The general inter-point distance between any point pair $\mathbf{x}_{i}$ and $\mathbf{x}_{j}$ can be expressed as follows:

$$
d_{i j}=d\left(\mathbf{x}_{i}, \mathbf{x}_{j}\right)=\left[\sum_{k=1}^{n_{v}}\left|x_{i k}-x_{j k}\right|^{t}\right]^{1 / t}, t=1 \text { or } 2
$$

In the present work, $p=50$ and $t=1$ are used following the suggestions of Jin et al. [25].

Table 1: Approaches for constructing the optimal Latin hypercube design.

\begin{tabular}{|l|l|l|l|}
\hline \hline Researchers & Year & Algorithm & Objective functions \\
\hline Audze and Eglājs [19] & 1977 & Coordinates Exchange Algorithm & Potential Energy \\
\hline Park [20] & 1994 & $\begin{array}{l}\text { A 2-stage (exchange- and Newton-type) } \\
\text { algorithm }\end{array}$ & $\begin{array}{l}\text { Integrated mean squared error and } \\
\text { entropy criteria }\end{array}$ \\
\hline Morris and Mitchell [21] & 1995 & Simulated annealing & $\phi_{p}$ criterion \\
\hline Ye et al. [22] & 2000 & Columnwise-pairwise & $\phi_{p}$ and entropy criteria \\
\hline Fang et al. [23] & 2002 & Threshold accepting algorithm & Centered $L_{2}$-discrepancy \\
\hline Bates et al. [24] & 2004 & Genetic algorithm & Potential energy \\
\hline Jin et al. [25] & 2005 & $\begin{array}{l}\text { Enhanced stochastic evolutionary } \\
\text { algorithm }\end{array}$ & $\begin{array}{l}\phi_{p} \text { criterion, entropy and } L_{2} \\
\text { discrepancy }\end{array}$ \\
\hline $\begin{array}{l}\text { Liefvendahl and Stocki } \\
\text { [26] }\end{array}$ & 2006 & $\begin{array}{l}\text { Columnwise-pairwise and genetic } \\
\text { algorithms }\end{array}$ & $\begin{array}{l}\text { Minimum distance and Audze- Eglājs } \\
\text { functions }\end{array}$ \\
\hline van Dam et al. [27] & 2007 & Branch-and-bound algorithm & 1-norm and infinite norm distances \\
\hline Grosso et al. [28] & 2008 & $\begin{array}{l}\text { Iterated local search and simulated } \\
\text { annealing algorithms }\end{array}$ & $\phi_{p}$ criterion \\
\hline \hline
\end{tabular}

Figure 1-(b) shows what point locations one can expect to obtain if the $\phi_{p}$ criterion is used to generate a LHD with $n_{v}=2$ and $n_{p}=16$ (although other criteria may also lead to the same distribution of points).

In this work, the Translational Propagation algorithm is presented for obtaining optimum or near optimum Latin hypercube designs. This algorithm requires minimal computational effort and does not use formal optimization. The aim is to solve the optimization problem in an approximate sense, i.e., to obtain a good Latin hypercube quickly, rather than finding the best possible solution. The algorithm exploits simple translation of small building blocks (consisting of one or more points) in the hyperspace. The obtained LHD is referred to as TPLHD (a Latin hypercube 
design obtained via the Translational Propagation algorithm). In general, the obtained TPLHD could be useful by themselves as optimum or near optimum Latin hypercube designs, or as an initial guess for numerical implementations based on some optimization algorithm.

The rest of the paper is organized as follows. Section II describes the translational propagation algorithm for obtaining Latin hypercube designs. Section III describes the numerical experiments used in this study. Section IV presents the results and discussion. Finally, the paper is closed by recapitulating salient points and concluding remarks.

\section{Latin Hypercube via Translational Propagation Algorithm}

\section{A. Basic Algorithm}

The proposed approach is based on the idea of constructing the optimal $n_{v}$-dimensional Latin hypercube design from a fairly small $n_{v}$-dimensional seed design. The simple example of a $16 \times 2$ (i.e., sixteen points in two dimensions) Latin hypercube design is used to explain the methodology. Figure 2 shows some possible twodimensional seed designs. While there is no limitation on the number of points for the seed design, the seed design can be as simple as just a single point $\left(1 \times n_{v}\right.$ design). For simplicity, the seed of Figure 2-(a) is seed used in the $16 \times 2$ Latin hypercube example.

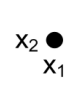

(a) $1 \times 2$ seed design.

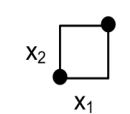

(b) $2 \times 2$ seed design.

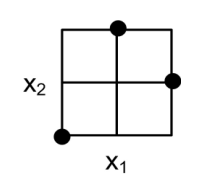

(c) $3 \times 2$ seed design.

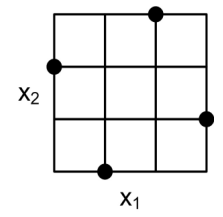

(d) $4 \times 2$ seed design.

\section{Figure 2: Example of seed designs for 2 variables.}

In order to construct a Latin hypercube design of $n_{p}$ points from a seed design of $n_{s}$ points, the design space is

first divide into a total of $n_{b}$ blocks such that:

$$
n_{b}=\frac{n_{p}}{n_{s}}
$$

Equation (3) means that each dimension is partitioned into the same number of divisions, $n_{d}$, calculated by: 


$$
n_{d}=\left(n_{b}\right)^{\frac{1}{n_{v}}}
$$

In the example of the $16 \times 2$ Latin hypercube design (i.e. $n_{p}=16$ and $n_{v}=2$ ), considering $n_{s}=1$ (seed design selected from Figure 2-(a)), one obtains $n_{b}=16$ and $n_{d}=4$ from Eqs. (3) and (4).

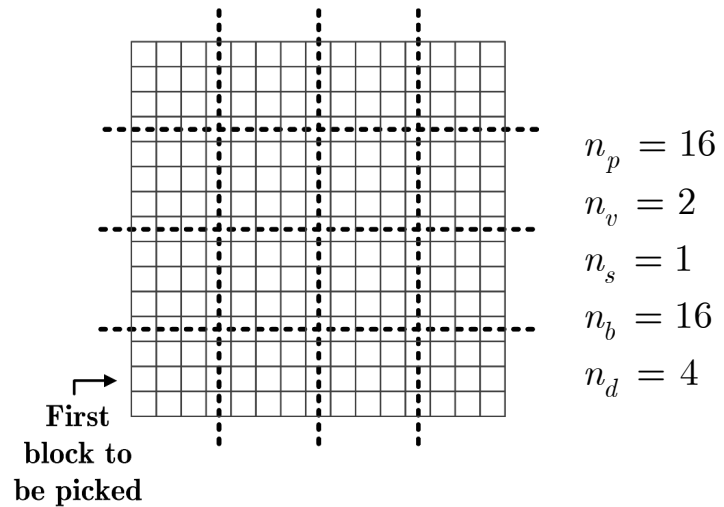

Figure 3: $16 \times 2$ Latin hypercube mesh divided into blocks (4 divisions in each dimension results in 16 blocks). The left-lower block is the first one to be picked in the algorithm.

Next, each block is filled with the previously selected seed design. Figure 3 shows the division of the design space for the $16 \times 2$ Latin hypercube design and which of the blocks is the first one to be picked in the algorithm. Figure 4 illustrates the process step by step. First, the seed design is properly scaled and placed at the origin of the first block, as shown in Figure 4-(a). Next, the block with the seed design is iteratively shifted by $n_{p} / n_{d}$ levels in one of the dimensions. Every time that the seed design is shifted, a new point is added to the experimental design. Figure 4-(b) shows the first shift of the seed design (chosen to be in the horizontal direction). To preserve the Latin hypercube property of only a single point per level; Figure 4-(b) shows that there also has to be a one-level shift in the vertical direction. In the general case, a displacement vector is built for each accounting for the shifting in the dimension of interest (horizontal direction in the example) as well a shift in all other dimensions to preserve the Latin hypercube properties (vertical direction in our example). The shifting process continues in one of the dimensions until all the divisions in this dimension are filled with the seed design as illustrated in Figure 4 (a-d). In the next step, the current set of points (newly filled division) is used as a new seed design and the procedure of 
shifting the seed design is repeated in the next dimension. Figure $4(\mathrm{e}-\mathrm{g})$ illustrates the shifting procedure in the vertical direction.

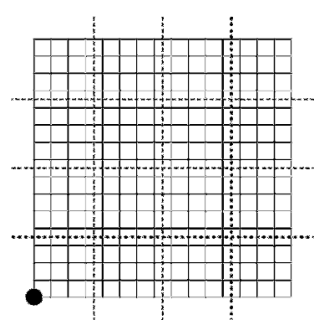

(a) Step 1

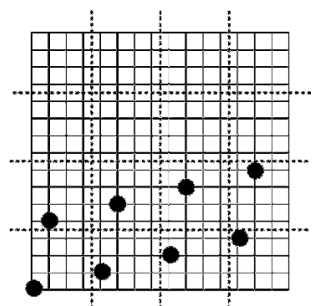

(e) Step 5

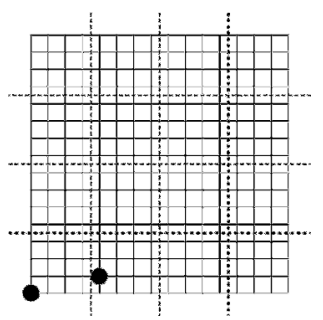

(b) Step 2

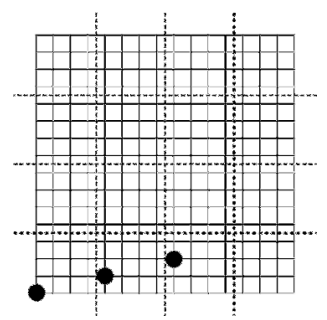

(c) Step 3

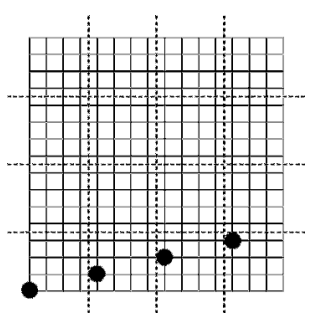

(d) Step 4

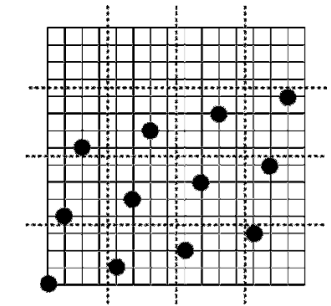

(f) Step 6

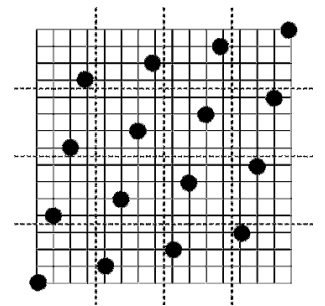

(g) Step 7

Figure 4: Process of creating the $16 \times 2$ enhanced Latin hypercube design. Figure 4-(a) illustrates the initial placement of the seed. Figure 4-(b) to (d) shows the translation of the seed in the horizontal direction (which is accompanied by a one-level vertical displacement to preserve Latin hypercube properties). Figure 4-(d) also represents the newly created "seed" that will be translated in the vertical direction. Figure 4-(e) to (g) illustrates the translation in the vertical direction (which is accompanied by horizontal displacement of one level).

The biggest advantage of this approach is that there are no calculations to perform. All operations can be viewed as a simple translation of the seed designs in the $n_{v}$-dimensional hypercube. Although efficient for generating large designs, the algorithm proposed up to now fails to provide flexibility for the total number of points in the final Latin hypercube design. The approach presented so far is limited in the sense that Eqs. (3) and (4) must hold. The next section describes a strategy to overcome this limitation and generate designs with arbitrary number of points.

\section{B. Generating experimental designs of any size}

To generate a Latin hypercube design with any number of points, the first step is to generate a TPLHD that has at least the required number of points using the algorithm described above. If this design contains the required number of points, the process is completed. Otherwise, an experimental design larger than the required is created 
and a resizing process is used to reduce the number of points to the desired one. The points are removed one-by-one from the initially created TPLHD by discarding the points that are the furthest from the center of the hypercube and reallocating remaining points to fill the whole design (preserving the Latin hypercube properties). In the proposed algorithm removing the points furthest from the center does not reduce the area of exploration. After removing the points, the final design is rescaled to cover the whole design space.

To illustrate the approach, consider the example where a $12 \times 2$ Latin hypercube is created using the one point seed of Figure 2-(a) (i.e., $n_{s}=1$ ). From Eqs. (3) and (4), $n_{d}=n_{p}^{1 / n_{v}} \cong 3.46$ does not result in an integer number in this case. Rounding $n_{d} \cong 3.46$ up would give $n_{d}=4$ and the next largest design that can be constructed is the $16 \times 2$, as illustrated in Figure 4. The resizing process begins with first calculating the distance between each of the sixteen points and the center of the design space. To create a $12 \times 2$ design out of a $16 \times 2$ one, the four points furthest from the center have to be removed. In practice, this means the points of the original TPLHD have to be ranked according to the distance from the center of the design space. The suggested resizing algorithm, computes these distances only one time, during the very first iteration. When a point is removed, the levels occupied by its projection along each of the dimensions have to be eliminated. This ensures the Latin hypercube property that only a single point is found at any of the levels. Figure 5 illustrates the resizing process step by step. The number of points in the design actually shrinks but the final design still represents samples over the same design space. Figure 5-(a) shows that in the $16 \times 2$ design the points in the left-bottom and right-top corners are equally far from the center. Due to symmetry, it is not important which of the points will be removed first. The top-right point is removed first, Figure 5-(a); and the bottom-left one is removed next, Figure 5-(b). Figure 5-(c) might be the best illustration of how the algorithm guarantees the Latin hypercube properties because it is the first time that an internal level is removed. Removing a point is as simple as eliminating it from the experimental design. However, as illustrated in Figure 6, this would leave empty levels (breaking the Latin hypercube requirements). The correct implementation of the resizing algorithm (see Figure 5-(c)) takes care of this limitation by also eliminating the levels that the point used to occupy. In Figure 5-(c) this means that the three points on the right would move to the left (occupying the empty level that was in between the points). Next, the remaining points are scaled to cover the original design space. The scaling is as simple as the mapping of the remaining points to the design space of interest such that the lower and upper bounds are sampled by one of the points of the design (i.e., simple unidirectional scaling of all points). After 
each step, a new near optimum Latin hypercube is obtained with one point less. The process continues until the $12 \times 2$ design is achieved. Removing points/levels part of the algorithm reduces the number of points of the experimental design, while preserving the Latin hypercube requirements. On the other hand, the resizing part makes the experimental design to fit in the original design space again.
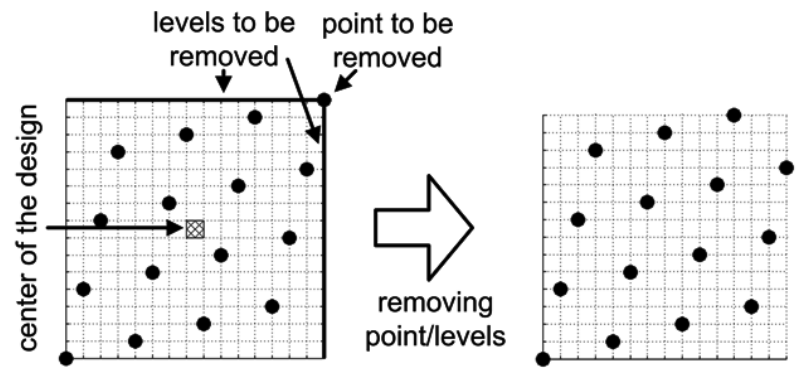

(a) Step 1.
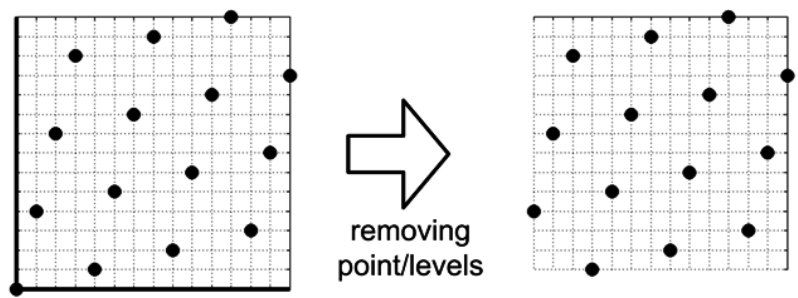

(b) Step 2 .
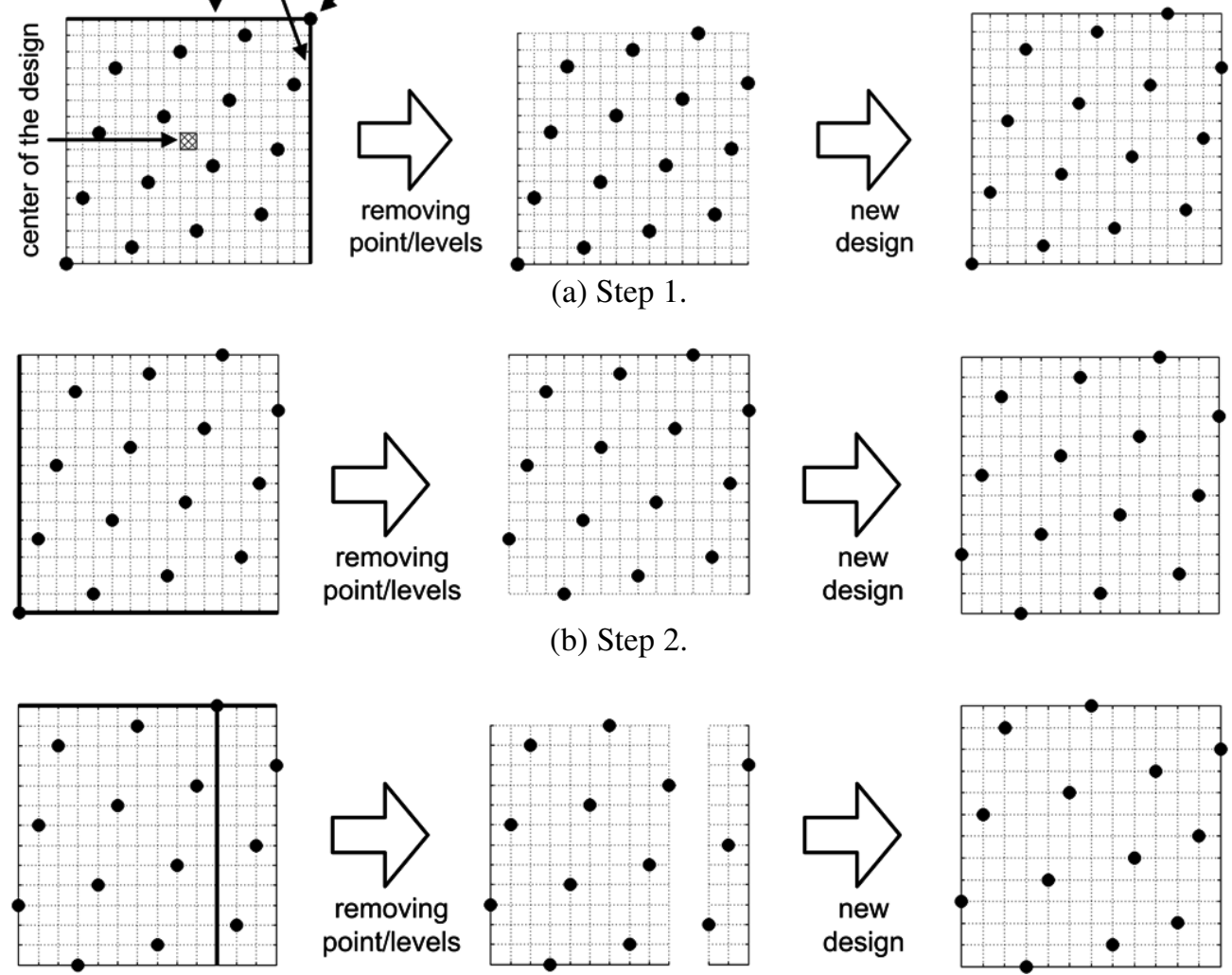

(c) Step 3
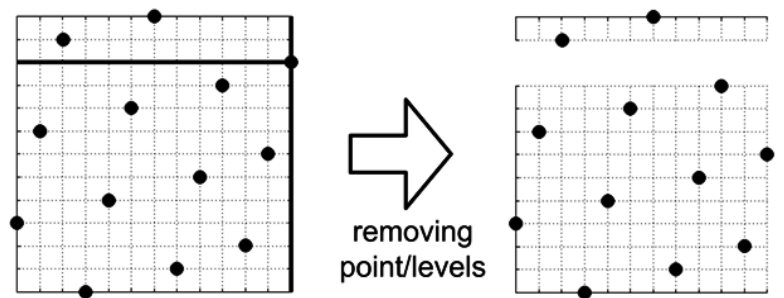

(d) Step 4.

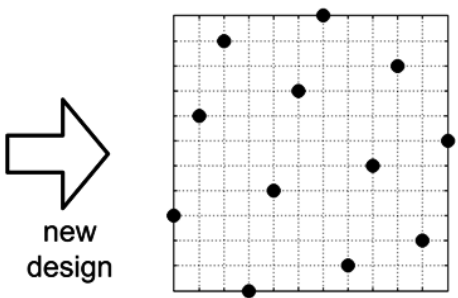

Figure 5: Process of creating a $12 \times 2$ enhanced Latin hypercube design from a $16 \times 2$ design.

\section{Seed designs}

Using the described algorithm, one can create different instances of the TPLHD by changing the seed design, as illustrated by Figure 7 for the $16 \times 2$ TPLHD. As it is not known beforehand which seed design will lead to the best 
design in terms of the $\phi_{p}$ criterion, one would not know what seed design to use. However, this might be a positive feature of the algorithm. First, it is possible that the different designs are comparably good (when looking at the $\phi_{p}$ criterion). Second, due to the low cost of the algorithm (as we will show in the next sections), it pays to create instances of the Latin hypercube with different seeds and then pick the best one according to the $\phi_{p}$ criterion.

Figure 6: Wrong step of the resizing algorithm.
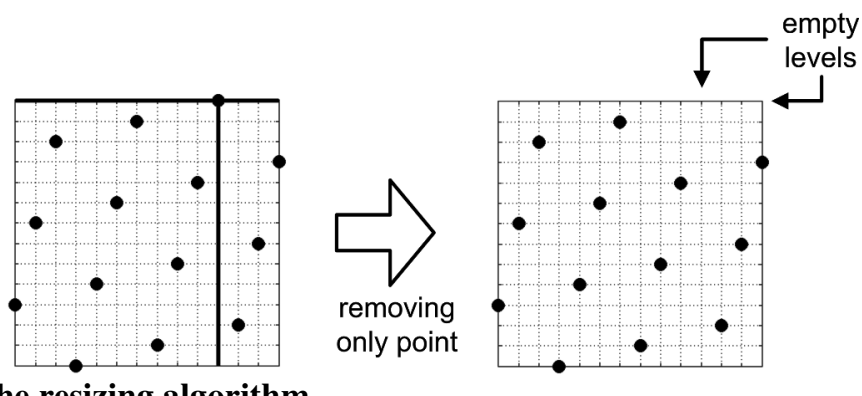

छ

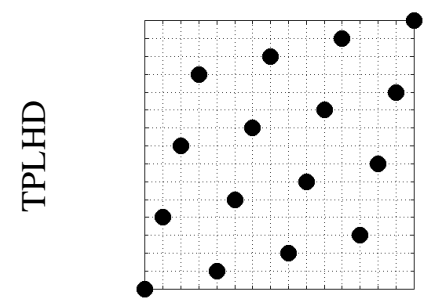

(a) From 1-point seed.
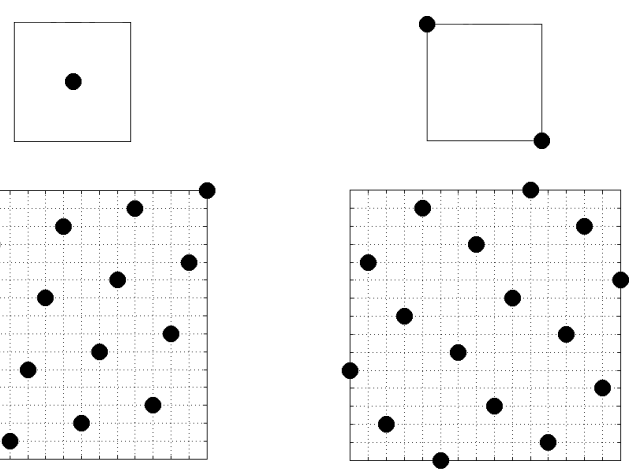

(b) From 2-points seed

Figure 7: $16 \times 2$ TPLHD obtained with different seeds.

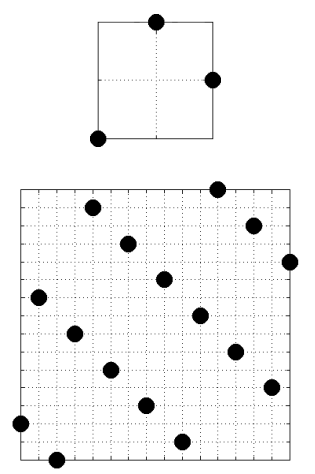

(c) From 3-points seed.

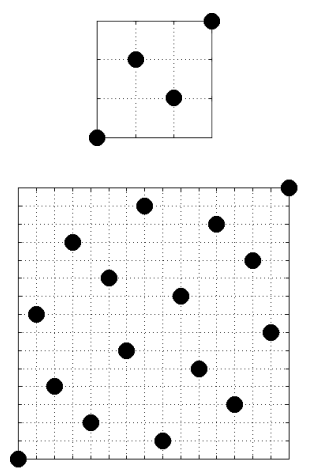

(d) From 4-points seed.

Because of the shifting process, the number of levels in a block is greater than the number of points in the seed design. This means that when creating design starting from seeds with more than one point $\left(n_{s}>1\right)$ it is necessary to reshape them to fit into one block of the TPLHD. Figure 8 illustrates this idea with the design of a $16 \times 2$ TPLHD starting from a $4 \times 2$ seed (i.e., $n_{s}=4$ ). Figure 8 -(a) shows the initial $4 \times 2$ seed (that respects the Latin hypercube properties). It is necessary to insert as many empty levels between two consecutive projections of points as the number of division in a given directions, as shown in Figure 8-(b). This way, at the end of the shifting 
process, there will be only one point per level (see Figure 8-(c)). The next section reviews the implementation of what was described so far.

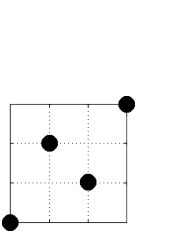

(a) Original seed.

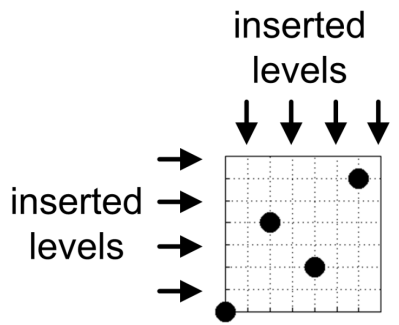

(b) Seed reshaped to fit in one block of the TPLHD.

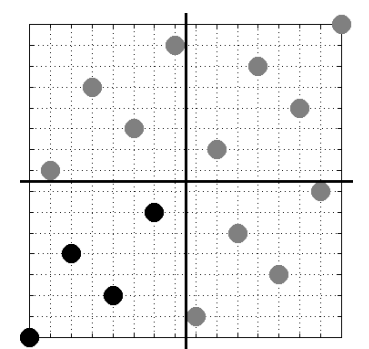

(c) Seed design filling different blocks of the TPLHD.

Figure 8: Illustration of the reshaping of the seed design.

\section{Summary of the algorithm}

The proposed algorithm is inspired by the optimization of the $\phi_{p}$ criterion penalizing designs with close points (due to $p=50$ and $t=1$ in Eq. (1)). Good Latin hypercube designs are expected to be obtained because the minimum distance is fixed at the level of seed design. This is particularly clear for large number of points in low dimensions. Figure 9 illustrates the Translational Propagation algorithm. The input parameters are the initial seed design $\mathbf{s}$, the number of points in the seed design, $n_{s}$, number of points of the desired Latin hypercube design, $n_{p}$, and the number of variables, $n_{v} \cdot n_{p}^{*}$ and $n_{d}^{*}$ are the number of points and number of divisions of the first TPLHD to be created. While $n_{p}$ can assume any value, $n_{p}^{*}$ is such that Eq. (3) returns an integer number of blocks $n_{b}$. The first step is to check whether or not a bigger experimental design is needed. The number of divisions, $n_{d}$, and its rounded up value, $n_{d}^{*}$, are compared. If $n_{d}^{*}>n_{d}$, Eqs. (3) and (4) does not hold and the number of points in the TPLHD to be created, $n_{p}^{*}$, is greater than the desired, $n_{p}$. Next, the seed design is reshaped to fit in one block of the TPLHD (Section II.C), and a TPLHD of $n_{p}^{*}$ points is created (Section II.A). Finally, if $n_{p}^{*}>n_{p}$, the TPLHD previously obtained is resized such that it will have $n_{p}$ points (Section II.B). $\mathbf{X}$ is the final TPLHD (with $n_{p}$ points).

Appendix A gives the MATLAB implementation of the different building blocks of the translational propagation algorithm. 


\section{Numerical Experiments}

To illustrate the effectiveness of the proposed TPLHD, a set of configurations that covers the typical application range of the LHD is considered. The experimental designs vary from only two to twelve variables as summarized in Table 2. For each number of variables, three cases representing small, medium and large number of design points were considered. The number of design points was calculated relative to the number of coefficients in a full polynomial model for the specified number of variables. The small designs have two times more points than the number of coefficients in a full quadratic polynomial model, while the large designs have twenty times more points than the number of coefficients in the same model. The medium designs have two times more points than the number of coefficients in a full cubic polynomial model.

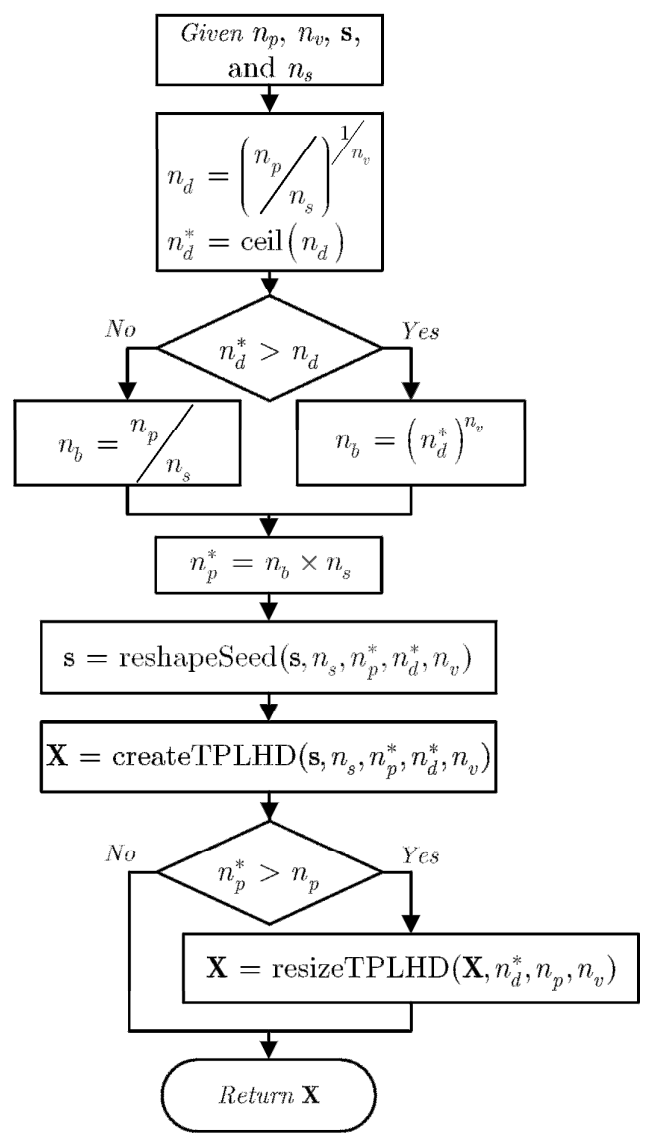

Figure 9: Flowchart of the Translational Propagation algorithm. 
Table 2: Latin hypercube design configurations considered.

\begin{tabular}{|c|c|c|c|}
\hline \hline \multirow{2}{*}{$\begin{array}{l}\text { No. of } \\
\text { variables }\end{array}$} & \multicolumn{3}{|c|}{ No. of points } \\
\cline { 2 - 4 } & Small designs & Medium designs & Large designs \\
\hline 2 & 12 & 20 & 120 \\
\hline 4 & 30 & 70 & 300 \\
\hline 6 & 56 & 168 & 560 \\
\hline 8 & 90 & 330 & 900 \\
\hline 10 & 132 & 572 & 1320 \\
\hline 12 & 182 & 910 & 1820 \\
\hline
\end{tabular}

The influence of the initial seed used to construct the TPLHD was studied first. For this study, different TPLHDs were generated using seeds with one to five points. The designs were then compared using the values of the $\phi_{p}$ criterion. Next, the efficiency of the proposed algorithm in approximating the optimal LHD was studied. For this study, an estimate of the range of the $\phi_{p}$ criterion for the LHDs proposed in Table 2 is created based on Monte Carlo simulation plus the results from 100 simulations of three different Latin Hypercube optimization techniques. The Monte Carlo simulation created 200,000 random LHDs for the configurations proposed in Table 2 (this is possible because the Latin hypercube algorithm allows for the creation of different designs based on a random number generator). The three different Latin Hypercube optimization techniques used in the study are:

1. The Enhanced Stochastic Evolutionary algorithm (ESEA) of Jin et al. [25]: set with maximum number of twenty iterations (but each simulation was allowed to run at most five iterations without improvement of the objective function).

2. The Genetic algorithm (GA) implementation of Bates et al. [24]: set with maximum number of fifty iterations (but each simulation was allowed to run at most twenty iterations without improvement of the objective function).

3. The native MATLAB function lhsdesign [30]: using the maxmin criterion (maximization of the minimum distance) and two hundred iterations. With these settings, the MATLAB lhsdesign function selects the best LHD from two hundred randomly created designs. The maxmin criterion is used to select the best design.

The settings for both GA and ESEA were based on a few trials for the design with 560 points and six variables. All simulations were conducted using an Intel Core 2 Quad CPU Q6600 at 2.40GHz, with 3GB of RAM running MATLAB 7.6 (R2008a) under Windows XP. The SURROGATES toolbox [29] was used to execute the TPLHD, ESEA, and GA algorithms under MATLAB [30]. 
Instead of showing only the $\phi_{p}$ criterion as defined in Eq. (1), a normalized version, $\tilde{\phi}_{p}$, is also presented for easier comparison:

$$
\tilde{\phi}_{p}=\frac{\phi_{p}-\min \left(\phi_{p}\right)}{\max \left(\phi_{p}\right)-\min \left(\phi_{p}\right)}
$$

where $\max \left(\phi_{p}\right)$ and $\min \left(\phi_{p}\right)$ are the maximum and minimum values of $\phi_{p}$ found in the generated DOEs (including both the TPLHDs and the Monte Carlo simulations); which means that $0 \leq \tilde{\phi}_{p} \leq 1$.

Using the Monte Carlo simulations and the optimization results, it is possible to estimate the range of variation of $\phi_{p}$ values and thus make a judgment if a specific design represents a substantial improvement or not. The $\tilde{\phi}_{p}$ criterion makes the comparison of the distributions of different designs easier. Although it eliminates the sense of absolute magnitude (because of the normalization), it permits identifying if the distributions are towards the low or high values of the $\phi_{p}$ criterion.

\section{Results and Discussion}

Table 3 shows the values of the $\phi_{p}$ criterion for TPLHDs obtained using different initial seed designs for design configurations of Table 2. From these results, it can be concluded that no single seed size is always the best (even considering designs with the same number of variables). For example, the single point seed is the best for the $12 \times 2$ and $20 \times 2$ designs, while the two-point seed is the best for the $120 \times 2$ design. However, there might be cases where different seeds are equally competitive. As illustration, this is the case for the $30 \times 4$ design, where the four-point and five-point seeds produce designs with nearly the same $\phi_{p}$ criterion. These two observations together with the fact that no time consuming optimization is required in the translational propagation algorithm suggests that it is possible to create several instances of the TPLHDs (based on different initial seeds) and select the design of choice based on the $\phi_{p}$ criterion. Table 3 also shows that an increase in dimensionality seems to favor the single point seed. The reason is that in higher dimensions a seed with multiple points acts like a cluster of points, hurting the $\phi_{p}$ criterion (this effect is less evident in lower dimensions). 
Table 3: $\phi_{p}$ criterion, defined in (1), ( $\left.p=50, t=1\right)$ for TPLHD constructed from different seeds (the best TPLHD is shown in bold face).

\begin{tabular}{|c|c|c|c|c|c|c|}
\hline \hline \multirow{2}{*}{$\begin{array}{l}\text { No. of } \\
\text { variables }\end{array}$} & \multirow{2}{*}{$\begin{array}{l}\text { No. of } \\
\text { points }\end{array}$} & \multicolumn{6}{|c|}{ Seed size } \\
\hline 2 & 12 & $\mathbf{2 . 8}$ & 2.9 & 3.8 & 3.7 & 3.8 \\
\hline 2 & 20 & $\mathbf{4}$ & 4.8 & 4.9 & 5 & $\mathbf{4}$ \\
\hline 2 & 120 & 11.0 & $\mathbf{9 . 4}$ & 12.6 & 13.4 & 13.8 \\
\hline 4 & 30 & 1.9 & 1.8 & 1.8 & $\mathbf{1 . 6}$ & 1.6 \\
\hline 4 & 70 & 2.7 & 5.0 & 2.9 & $\mathbf{2 . 0}$ & 2.1 \\
\hline 4 & 300 & 7.2 & 13.6 & 6.2 & $\mathbf{3 . 6}$ & 4.0 \\
\hline 6 & 56 & $\mathbf{1 . 7}$ & 2.1 & 1.8 & 3.7 & 1.7 \\
\hline 6 & 168 & 3.1 & 6.0 & $\mathbf{2 . 4}$ & 2.9 & $\mathbf{2 . 5}$ \\
\hline 6 & 560 & $\mathbf{3 . 2}$ & 11.7 & 5.3 & 7.9 & 3.6 \\
\hline 8 & 90 & $\mathbf{1 . 6}$ & 3.4 & 2.6 & 5.9 & 1.9 \\
\hline 8 & 330 & 3.7 & 4.6 & 3.1 & 3.0 & $\mathbf{2 . 5}$ \\
\hline 8 & 900 & 4.7 & 16.4 & 4.6 & 2.7 & $\mathbf{2 . 6}$ \\
\hline 10 & 132 & $\mathbf{1 . 6}$ & 3.7 & 3.9 & 6.6 & 3.5 \\
\hline 10 & 572 & $\mathbf{2 . 0}$ & 8.8 & 4.0 & 3.1 & 2.9 \\
\hline 10 & 1320 & 4.2 & 6.1 & 3.5 & 3.9 & $\mathbf{3 . 1}$ \\
\hline 12 & 182 & $\mathbf{1 . 7}$ & 11.3 & 3.1 & 1.9 & 2.6 \\
\hline 12 & 910 & $\mathbf{2 . 0}$ & 16.8 & 3.2 & 3.1 & 2.4 \\
\hline 12 & 1820 & $\mathbf{2 . 1}$ & 17.8 & 3.7 & 3.4 & 2.4 \\
\hline \hline
\end{tabular}

Table 4 shows detailed statistics regarding the $\phi_{p}$ criterion with data obtained during the Monte Carlo and optimization simulations (the 5th percentile is added to give information about the lower values of $\phi_{p}$ ). For up to six variables, TPLHD delivers exceptionally good designs (within five percent of the best designs for most of the cases). For eight to twelve variables, TPLHD is not attractive anymore (with designs located within the last quartile of the $\phi_{p}$ criterion distribution). The reader will notice that for ten variables the maximum value of $\phi_{p}$ drops when coming from 572 to 1320 points (contrarily from the trend of the other design configurations). This is because it is difficult to estimate this value for high dimensional cases (even with total of more than 200,000 simulations). On the other hand, the percentile information establishes the trend of increasing $\phi_{p}$ with the number of points. Since the algorithm was heuristically developed based on the visual observation of the patterns generated by the optimal LHD in two and three dimensions according to the $\phi_{p}$ criterion, it is of no surprise that the efficiency is harmed when the dimensionality increases. The range of the $\phi_{p}$ criterion varies with different design configurations and can also be very large (for example, the range for the $120 \times 2$ design is 52.9). Thus, only the normalized $\phi_{p}$ criterion, $\tilde{\phi}_{p}$, is used from this point on. 
Table 4: TPLHD and statistics about the $\phi_{p}$ criterion, defined in (1), $(p=50, t=1)$ for each studied configuration. Bold faces show when TPLHD offers the best design. TPLHD performs very well up to 6 dimensions.

\begin{tabular}{|c|c|c|c|c|c|c|c|c|c|}
\hline \multirow{2}{*}{$\begin{array}{c}\text { No. of } \\
\text { variables }\end{array}$} & \multirow{2}{*}{$\begin{array}{c}\text { No. of } \\
\text { points }\end{array}$} & \multirow{2}{*}{ TPLHD } & \multirow{2}{*}{ Min } & \multicolumn{7}{|c|}{ Percentiles } & \multirow{2}{*}{ Max } & \multirow{2}{*}{ Range } \\
\hline & 12 & $\mathbf{2 . 8}$ & 2.3 & 3.7 & 5.5 & 5.6 & 5.6 & 5.7 & 3.4 \\
\hline 2 & 20 & $\mathbf{4 . 0}$ & 3.4 & 6.4 & 9.5 & 9.6 & 9.7 & 10.0 & 6.6 \\
\hline 2 & 120 & $\mathbf{9 . 4}$ & 9.4 & 40.2 & 59.5 & 60.3 & 60.8 & 62.3 & 52.9 \\
\hline 2 & 30 & $\mathbf{1 . 6}$ & 1.5 & 2.3 & 2.9 & 3.2 & 3.7 & 7.4 & 5.9 \\
\hline 4 & 70 & $\mathbf{2 . 0}$ & 2.0 & 3.8 & 4.9 & 5.8 & 6.9 & 17.3 & 15.3 \\
\hline 4 & 300 & $\mathbf{3 . 6}$ & 3.4 & 8.6 & 11.1 & 13.0 & 15.7 & 74.8 & 71.3 \\
\hline 4 & 56 & $\mathbf{1 . 7}$ & 1.0 & 1.5 & 1.8 & 2.0 & 2.3 & 7.9 & 6.9 \\
\hline 6 & 168 & $\mathbf{2 . 4}$ & 1.3 & 2.4 & 2.8 & 3.2 & 3.6 & 23.9 & 22.6 \\
\hline 6 & 560 & $\mathbf{3 . 2}$ & 1.8 & 3.7 & 4.4 & 4.9 & 5.7 & 32.9 & 31.1 \\
\hline 6 & 90 & 1.6 & 0.9 & 1.1 & 1.2 & 1.3 & 1.5 & 5.9 & 5.0 \\
\hline 8 & 330 & 2.5 & 1.4 & 1.6 & 1.8 & 2.0 & 2.2 & 9.7 & 8.3 \\
\hline 8 & 900 & 2.6 & 1.8 & 2.1 & 2.4 & 2.7 & 3.0 & 16.3 & 14.5 \\
\hline 8 & 132 & 1.6 & 0.7 & 0.8 & 0.9 & 1.0 & 1.1 & 6.6 & 5.8 \\
\hline 10 & 572 & 2.0 & 1.0 & 1.2 & 1.3 & 1.4 & 1.5 & 8.8 & 7.8 \\
\hline 10 & 1320 & 3.1 & 1.3 & 1.4 & 1.6 & 1.7 & 1.9 & 6.1 & 4.8 \\
\hline 10 & 182 & 1.7 & 0.6 & 0.7 & 0.7 & 0.8 & 0.8 & 11.3 & 10.7 \\
\hline 12 & 910 & 2.0 & 0.8 & 0.9 & 1.0 & 1.1 & 1.1 & 16.8 & 16.0 \\
\hline 12 & 1820 & 2.1 & 0.9 & 1.0 & 1.1 & 1.2 & 1.3 & 17.8 & 16.9 \\
\hline 12 & & & & & & & & & \\
\hline \hline
\end{tabular}

Figure 10 illustrates the box plots of the $\tilde{\phi}_{p}$ criterion for each of the studied configurations. A box plot shows the full data range on the y-axis. A box is defined by lines at the lower quartile (25\%), median (50\%), and upper quartile $(75 \%)$ values. Lines extend from each end of the box for a distance of 1.5 times the inter-quartile range or until the data limit is reached. The data points falling outside this 1.5 times the inter-quartile range represent outliers that are shown using "+" symbols. Figure 10 shows that the distribution of $\tilde{\phi}_{p}$ tends to lower values as the dimensionality of the problem grows. It does not necessarily mean that the LHD fills the space better; after all, the sparsity of the points severely increases with the number of variables. However, this behavior suggests that the probability of generating a design in the lower levels of the $\tilde{\phi}_{p}$ increases with the dimensionality. The following conclusions can thus be drawn from the numerical experimentation:

- In low dimensions: the optimum LHD is an outlier located in the lower region of the $\phi_{p}$ values; making it difficult to be obtained. 
- In high dimensions: most of the LH designs are in the lower region of the $\tilde{\phi}_{p}$; making it difficult to distinguish the optimal LHD (obviously according to this criterion only) and other several good representations of the LHD.

Figure 10 makes clear that the TPLHD represents a better approximation of the optimum LHD in low to medium dimensions (up to six variables) and thus we will focus only on the designs with up to six variables.

Table 5 allows a comparison between the TPLHD and the mean out of 100 simulations of three different optimization techniques: (i) the Enhanced Stochastic Evolutionary algorithm (ESEA) of Jin et al. [25]; (ii) the Genetic algorithm (GA) implementation of Bates et al. [24]; and (iii) the native MATLAB function Ihsdesign [30]. TPLHD was obtained by generating five candidates from different seeds and then picking the best one according to the $\phi_{p}$ criterion (which means that Table 5 shows the time needed to generate all five candidates. Appendix B shows the discussion on the number of points that needs to be allocated in each of the cases; which directly impacts the computational cost). Results in Table 5 reinforce that TPLHD offers very good solutions; with most of the designs presenting $\tilde{\phi}_{p}=0$. This means that TPLHD found the best result of the set of all simulations (including the Monte Carlo and all optimization ones). As for the computational cost, the TPLHD design is superior in most of the cases, or in the worst case matches the best time of the traditional optimization techniques. Clearly, the point density has a dramatic impact on the optimization techniques (especially ESEA and GA). For instance, for the ESEA with six variables, moving from 56 to 560 points makes the computational cost change from two seconds to a little more than 18 minutes. The lhsdesign MATLAB function tends to suffer less, since it can be seen as a Monte Carlo simulation with only 200 samples (200 is the number of iterations that lhsdesign was allowed to run). Still, in six dimensions, when moving from 56 to 560 points the computational cost of the lhsdesign increases more than 100 times. 


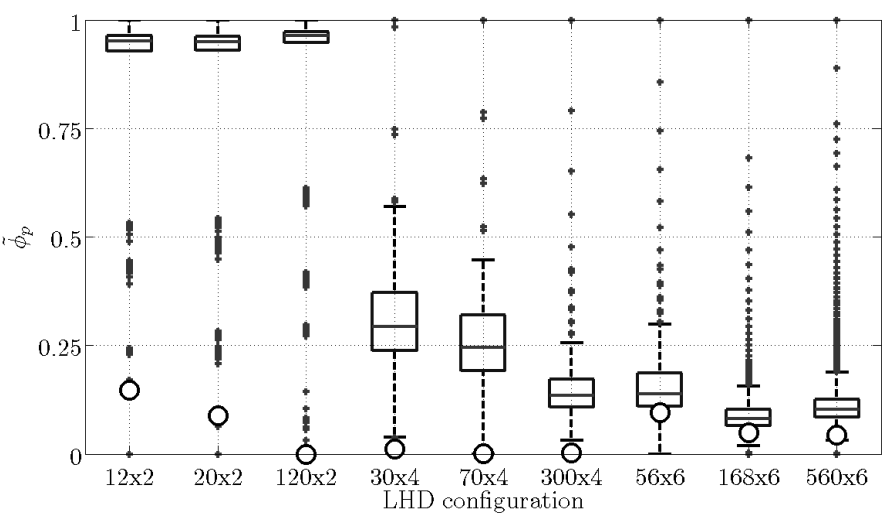

(a) Low to medium dimensions.

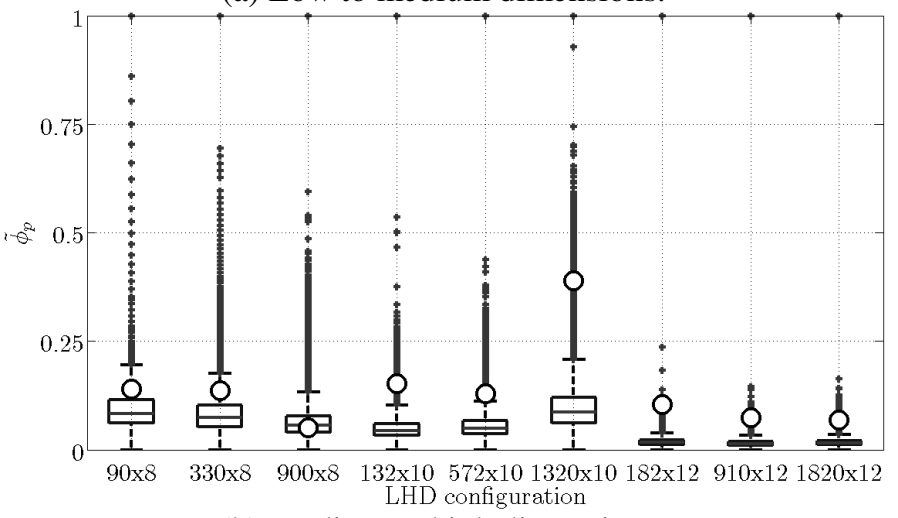

(b) Medium to high dimensions.

Figure 10: Boxplots of the $\tilde{\phi}_{p}$ criterion, defined in (5), $(p=50, t=1)$, where circles indicate the best

TPLHD. TPLHD represents a good solution in low to medium dimensions. $\tilde{\phi}_{p}$ ranges from 0 to 1.

Table 5 gives the impression that increasing the number of variables (no matter the point density) made the three optimization techniques to improve their own performance (in terms of the $\tilde{\phi}_{p}$ criterion). Considering the $\tilde{\phi}_{p}$ criterion of the designs with four and six variables, TPLHD and any of the optimization techniques would offer designs that are below 0.1. Then, the computational cost would leave only TPLHD and the lhsdesign as competing strategies. However, a closer look at the distributions of the $\tilde{\phi}_{p}$ criterion shows that TPLHD is the best choice.

Figure 11 contrasts the box plot of the $\tilde{\phi}_{p}$ criterion with the values found by TPLHD and the 0.1 threshold of Ihsdesign. Because the distributions tends to lower values, the 0.1 threshold is a bad value for the $300 \times 4$ design and is a marginal to undesired value in 6 dimensions. It is clear that TPLHD offers a better design (not mentioning a faster solution). 
Table 5: Performance comparison between TPLHD and the median out of 100 simulations of different optimization techniques. ESEA is the implementation of the Enhanced Stocastic Evolutionary algorithm of Jin et al. [25]. Ihsdesign is a native MATLAB function. GA refers to the Genetic algorithm implementation of Bates et al. [24]. $\tilde{\phi}_{p}$, defined in (5), ranges from 0 to 1.

\begin{tabular}{|c|c|c|c|c|c|c|c|c|c|c|}
\hline \hline \multicolumn{2}{|c|}{ No. of variables } & \multicolumn{3}{c|}{2} & \multicolumn{2}{c|}{4} & \multicolumn{3}{c|}{6} \\
\hline \multicolumn{2}{|c|}{ No. of points } & 12 & 20 & 120 & 30 & 70 & 300 & 56 & 168 & 560 \\
\hline \multirow{4}{*}{$\tilde{\phi}_{p}$} & TPLHD & 0.1 & 0.1 & 0 & 0 & 0 & 0 & 0.1 & 0 & 0 \\
\cline { 2 - 12 } & ESEA & 0.2 & 0.1 & 0.1 & 0 & 0 & 0 & 0 & 0 & 0 \\
\cline { 2 - 11 } & GA & 0.2 & 0.3 & 0.3 & 0.1 & 0.1 & 0 & 0 & 0 & 0 \\
\cline { 2 - 11 } & lhsdesign & 0.5 & 0.5 & 0.6 & 0.1 & 0.1 & 0.1 & 0.1 & 0. & 0.1 \\
\hline \multirow{3}{*}{$\begin{array}{c}\text { Time } \\
\text { (sec) }\end{array}$} & TPLHD & $\cong 0$ & $\cong 0$ & 0.1 & $\cong 0$ & $\cong 0$ & 0.7 & $\cong 0$ & 0.5 & 2 \\
\cline { 2 - 11 } & ESEA & $\cong 0$ & 0.2 & 13 & 0.3 & 3 & 173 & 2 & 34 & 1096 \\
\cline { 2 - 11 } & GA & 0.4 & 0.9 & 24 & 4 & 17 & 275 & 19 & 143 & 1509 \\
\cline { 2 - 11 } & lhsdesign & $\cong 0$ & $\cong 0$ & 0.2 & $\cong 0$ & 0.1 & 1.8 & 0.1 & 1.0 & 13 \\
\hline \hline
\end{tabular}

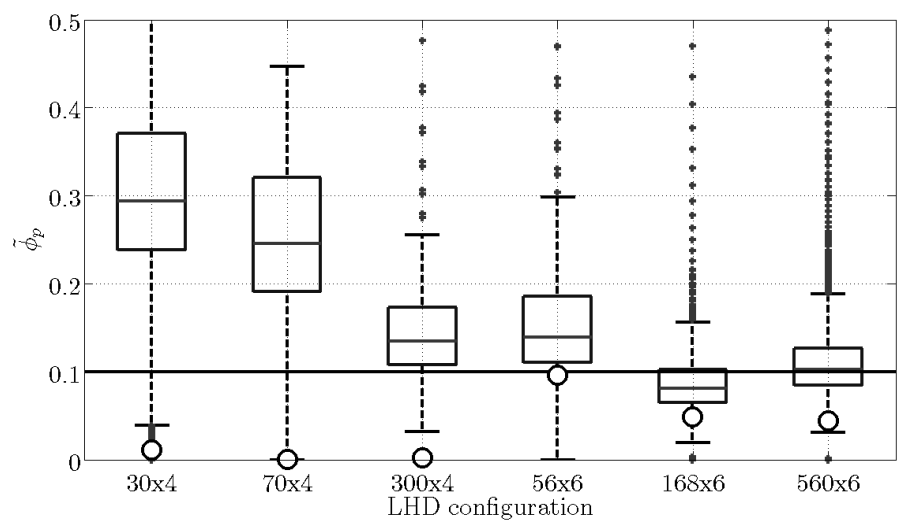

Figure 11: Boxplots of the $\tilde{\phi}_{p}$ criterion between 0 and 0.5 for the four and six dimensional designs. $\tilde{\phi}_{p}$ is defined in (5), ( $p=50, t=1)$ and ranges from 0 to 1 . Circles indicate the TPLHD.

One of the reasons for the poor performance of the TPLHD in high dimensions might be the existence of the directionality property of TPLHD. Because of the way the points are created in TPLHD, they tend to be stretched along one direction. Even the optimal LHD (obtained with ESEA or GA for example) from Figure 1-(b) may be considered as having points placed in a preferred direction (close to 45 degrees). Goel et al. [14] discussed that a single criterion for generating design of experiments may lead to large deteriorations in other criteria. In the proposed algorithm, the $\phi_{p}$ criterion is used and the directionality of the resulting Latin hypercube designs (for both the TPLHD and the designs obtained with traditional optimization) is a clear loss. There has been work on the optimization of the space-filling properties of the LHD while preserving low correlation between variables. Cioppa and Lucas [31] presented an algorithm that improves the space-filling properties of LHD at the expense of inducing small correlations between the columns in the design matrix. However, authors warned that the approach is 
computationally prohibitive. Hernandez [32] presented an extensive study on a set of methodologies to create design matrices with little or no correlation (including saturated nearly orthogonal LHDs). Franco et al. [33] discussed a radar-shaped statistic for identifying the directionality property in a DOE (especially efficient in low dimension). However there has been little research on how to employ this statistic for improving DOE and not just for identifying the directionality in an existing DOE, the directionality statistic was not employed to improve existing DOE in this paper. Nevertheless, the focus of this research is the translational propagation algorithm as a cheap alternative to traditional optimization of the Latin hypercube designs based on the $\phi_{p}$ criterion.

Other, more general questions arise in higher dimensions: it is not clear what is the meaning of space-filling designs and the relative cost of any optimization strategy of experimental designs. The sparsity of the data may make it difficult to judge whether designs given by the optimization of the Latin hypercube are in fact space-filling designs. Even if they are, considering plots such as those in Figure 10, the computational cost of strategies like TPLHD may end up being very close to random search (such as in lhsdesign). It may happen that in higher dimensions it is not worth investing much in time consuming optimization (few iterations of random search may provide satisfactory results). In spite of that, authors intend to investigate the potential of the radar-shaped statistic in to improve the TPLHD properties.

\section{Conclusions}

A methodology for creating Latin hypercube designs via translational propagation algorithm (TPLHD) is proposed. The approach is based on the idea that a simple "seed design" with a few points can be used as a building block to construct a near optimal Latin Hypercube design (LHD). The main advantage of the proposed methodology is that it requires virtually no computational time. The approach was tested on eighteen different experimental design configurations with varying dimensionality and point density. Monte Carlo simulations were used to support the analysis of the algorithm's performance. For these cases it was found that:

- Based on the Monte Carlo simulations, the probability of randomly generating good designs in terms of the $\phi_{p}$ criterion (i.e. designs with low values of $\phi_{p}$ ) increases with the dimensionality. This does not mean that the designs will fill the space better, it only means that designs tends to be equally competitive. This fact makes computationally cheap strategies such as random search attractive in high dimensions. 
- It was found that for the low dimensional cases (up to six variables) the TPLHD approximates the optimal LHD (using $\phi_{p}$ criterion) very well. In higher dimensions (eight to twelve variables), the TPLHD does not approximate the optimum solution well anymore.

As the time for generating the TPLHD is very small, in small to medium dimensions it is recommended to generate several TPLHDs (using seed designs with different number of points) and to pick the best one according to the $\phi_{p}$ criterion.

\section{Acknowledgments}

Authors would like to thank everybody at Vanderplaats Research and Development, Inc. (VR\&D) for the encouragement and support. This work was started when authors were employed by VR\&D in various positions.

\section{Appendix}

\section{A. MATLAB implementation of the Translational Propagation algorithm}

Table 6 to Table 9 give the MATLAB code of our implementation of the translational propagation algorithm. For simplicity, the levels of the Latin hypercube are represented by integer values. This means that each of the points of the created Latin hypercube design can assume values from one to the number of points. The created Latin hypercube needs to be properly scaled to the design space of interest. Table 6 gives the main function; which has as input parameters the number of points, $n_{p}$, and variables, $n_{v}$, of the desired Latin hypercube design, as well as the seed design, $\mathbf{s}$, and its number of points, $n_{s}$. The points in the seed design initially assume values from one to the $n_{s}$. Lines 9 to 17 of the algorithm shown in Table 6 illustrate how to take care of the size of the Latin hypercube to be created using the translational propagation algorithm. At first, a Latin hypercube observing Eqs. (3) and (4) is created; even if it means creating a design bigger than required. The seed design is then properly placed into the first block of to be filled by the algorithm (line 19). Line 43 is the call for the function that creates the initial Latin hypercube design. If necessary, the design is then resized to the initially set configuration. Table 7 gives the implementation of the reshaping of the seed design (see Section II.C). Table 8 illustrates the body of the translational propagation algorithm. Lines 16 to 18 show how to create the displacement vector that is used to translate the seed in the hyperspace. Finally, Table 9 presents the implementation of the resizing process. Lines 8 to 
15 show how we reduce the initially large Latin hypercube to the $n_{p}$ initially set. Lines 18 to 30 present how to restore the Latin hypercube condition of one point per level to the remaining points.

Table 6: MATLAB implementation of the translational propagation algorithm. The created design ranges from one to the number of points. The algorithm works with integer indexes for each point. ceil $(x)$ is the MATLAB function that rounds the elements of $x$ up to the nearest integers. ones $(m, n)$ is the MATLAB function that creates an $m$-by- $n$ matrix of ones. See Table 7 for details about reshapeSeed. See Table 8 for details about createTPLHD. See Table 9 for details about resizeTPLHD. vertcat $(A, B)$ is the MATLAB function that performs the vertical concatenation of matrices $A$ and $B$.

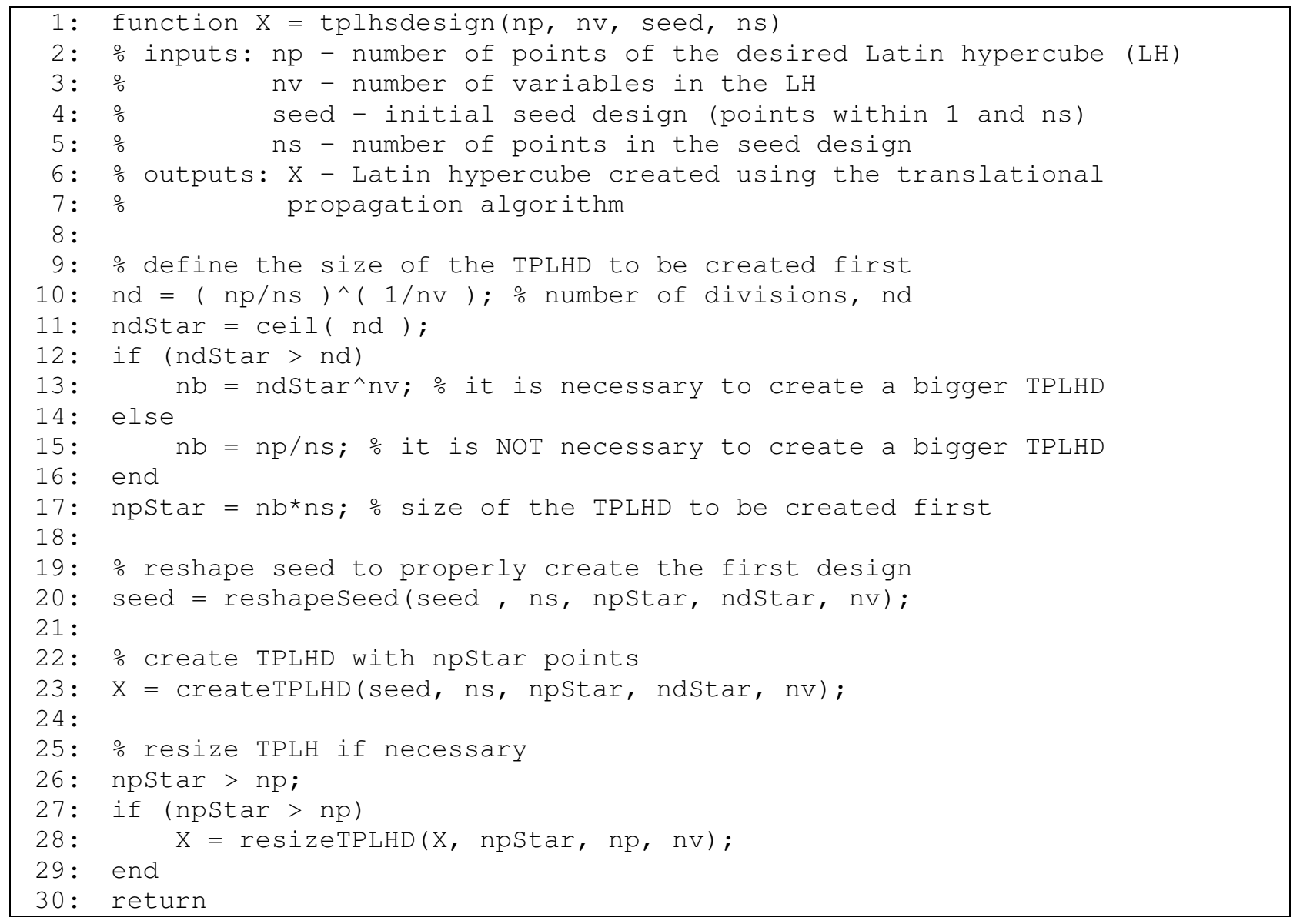


Table 7: MATLAB implementation of the reshapeseed function. ones $(m, n)$ is the MATLAB function that creates an $m$-by- $n$ matrix of ones. $\operatorname{round}(\mathbf{x})$ is the MATLAB function that rounds the elements of $\mathbf{X}$ to the nearest integers.

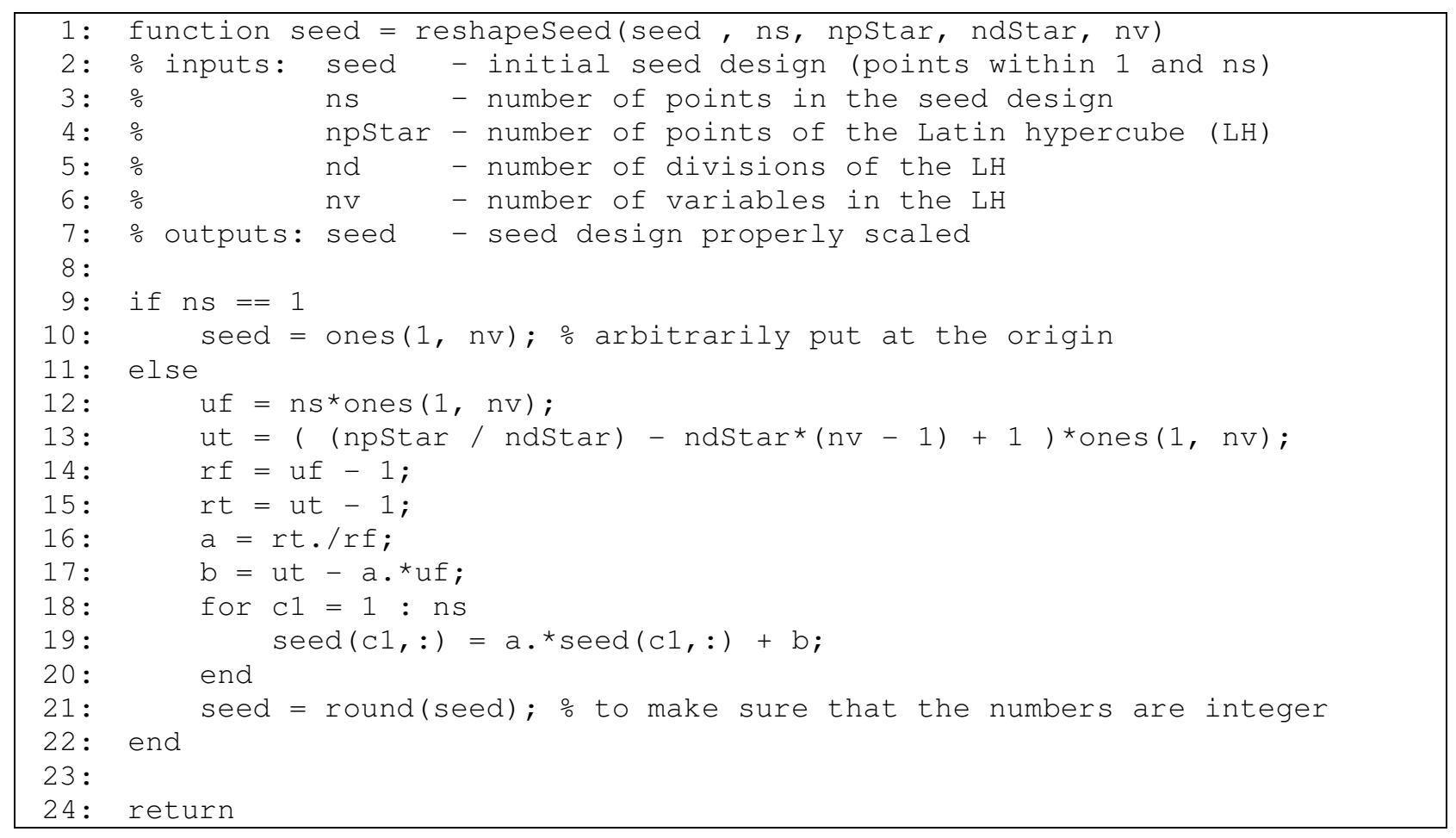


Table 8: MATLAB implementation of the createTPLHD function. ones $(m, n)$ is the MATLAB function that creates an $m$-by- $n$ matrix of ones. length $(\mathbf{a})$ is the MATLAB function that returns the number of entries in the vector $a$. $\operatorname{vertcat}(A, B)$ is the MATLAB function that does the vertical concatenation of the matrices $A$ and $B$.

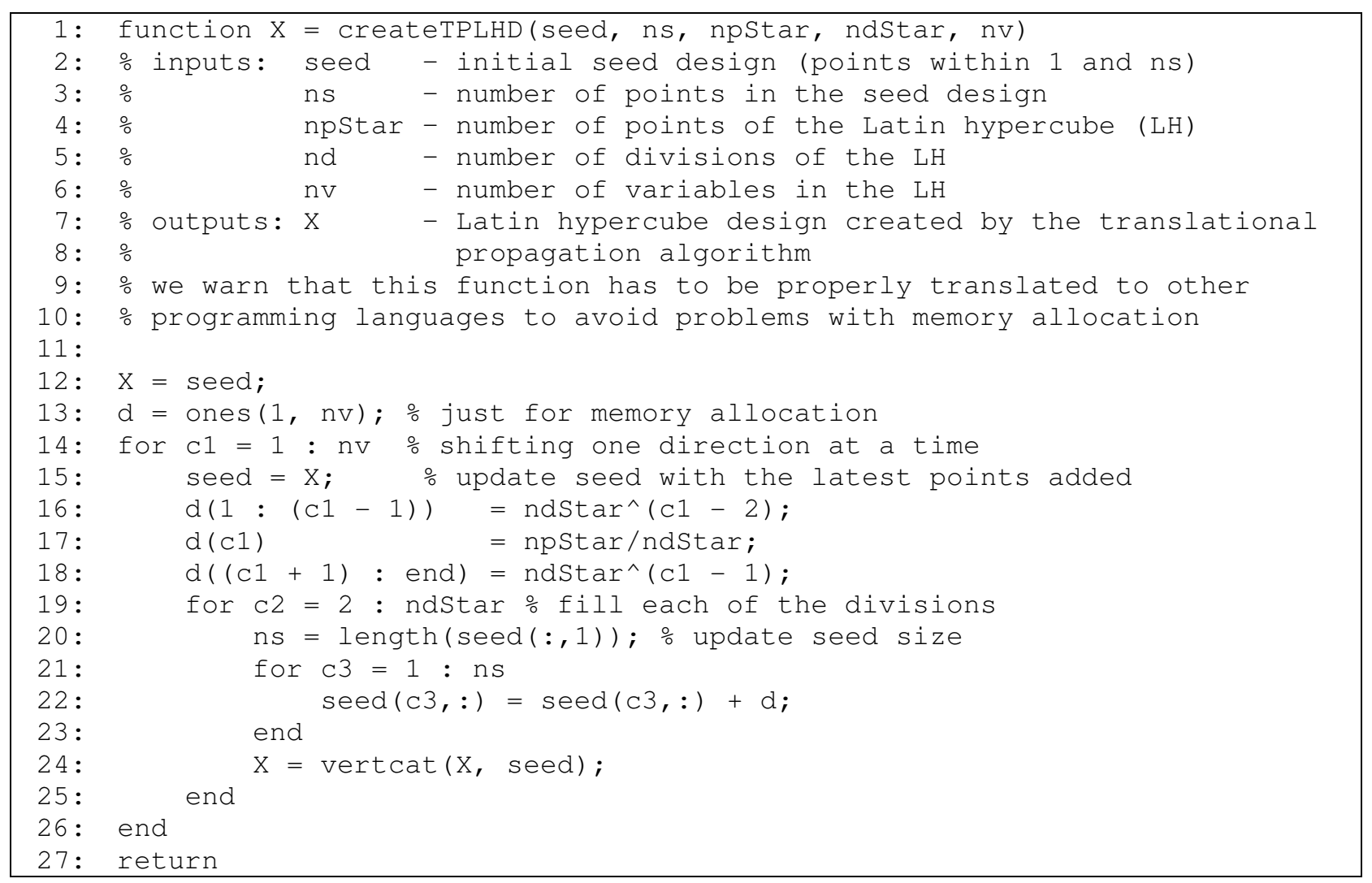


Table 9: MATLAB implementation of the resizeTPLHD function. ones $(m, n)$ is the MATLAB function that creates an $m$-by- $n$ matrix of ones. $\operatorname{zeros}(m, n)$ is the MATLAB function that creates an $m$-by- $n$ matrix of zeros. $\operatorname{norm}(\mathbf{x})$ is the MATLAB function that computes the vector norm $\left(\sqrt{\sum\left(x_{i}^{2}\right)}\right) \cdot \min (\mathbf{x})$ is the MATLAB function that finds the smallest element in $x$. $\operatorname{sort}(a)$ is the MATLAB function that sorts the vector $\mathbf{a}$ in the ascending order, returning the sorted and the index vectors. $\operatorname{sortrows}(\mathrm{X}, \mathrm{COL})$ is the MATLAB function that sorts the matrix $X$ based on the columns specified in the vector $C O L$. isequal $(\mathrm{A}, \mathrm{B})$ is the MATLAB function that returns logical 1 (TRUE) if arrays A and $\mathrm{B}$ are the same size and contain the same values, and logical 0 (FALSE) otherwise.

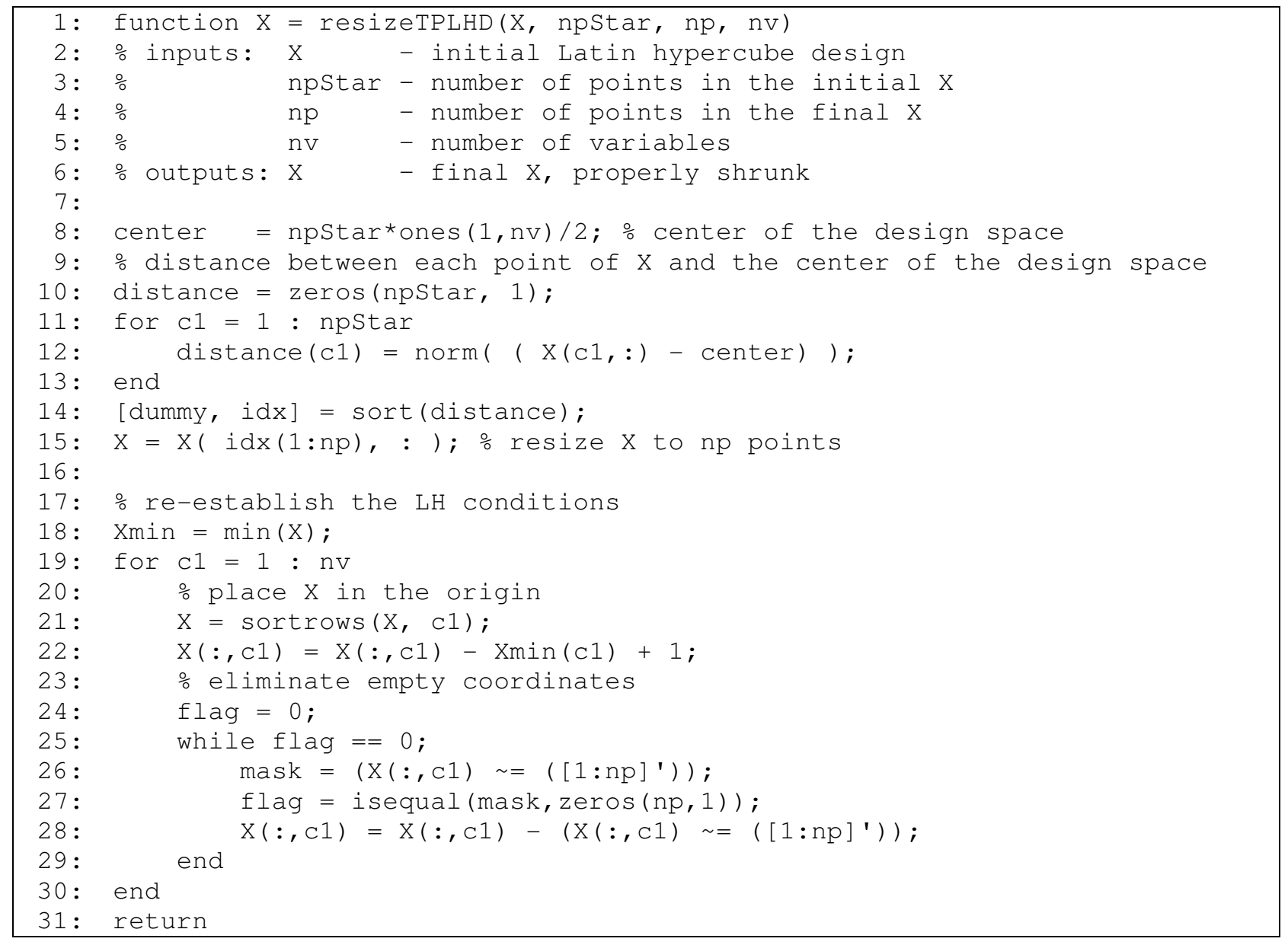




\section{B. Number of points allocated before resizing the TPLHD}

As discussed in Section II, the number of points that needs to be allocated for a given configuration of the TPLHD is dictated by Eqs. (3) and (4). In the most general cases, a resizing process needs to be used. Thus, the total number of points initially generated dictates the computational cost of the TPLHD algorithm. Table 10 shows the number of points allocated before the resizing process for each of the configurations studied in this paper. It can be observed that the more variables, the more points are discarded. This confirms the fact that design strategies based on the domain subdivision (the translational propagation algorithm is an example) tend to have poor performance as dimensionality increases.

Table 10: Size of the TPLHD created before the resizing process for each seed size. The final best TPLHD is shown in bold face.

\begin{tabular}{|c|c|c|c|c|c|c|}
\hline \hline \multirow{2}{*}{$\begin{array}{l}\text { No. of } \\
\text { variables }\end{array}$} & \multirow{2}{*}{$\begin{array}{l}\text { No. of } \\
\text { points }\end{array}$} & \multicolumn{6}{|c|}{ Seed size } \\
\hline 2 & 1 & 2 & 3 & 4 & 5 \\
\hline 2 & 20 & $\mathbf{1 6}$ & 18 & 12 & 16 & 20 \\
\hline 2 & 120 & 121 & $\mathbf{1 2 8}$ & 147 & 144 & 125 \\
\hline 4 & 30 & 81 & 32 & 48 & $\mathbf{6 4}$ & 80 \\
\hline 4 & 70 & 81 & 162 & 243 & $\mathbf{3 2 4}$ & $\mathbf{8 0}$ \\
\hline 4 & 300 & 625 & 512 & 768 & $\mathbf{3 2 4}$ & 405 \\
\hline 6 & 56 & $\mathbf{6 4}$ & 128 & 192 & 256 & 320 \\
\hline 6 & 168 & 729 & 1458 & $\mathbf{1 9 2}$ & 256 & 320 \\
\hline 6 & 560 & $\mathbf{7 2 9}$ & 1458 & 2187 & 2916 & 3645 \\
\hline \hline
\end{tabular}

\section{References}

[1] Venkataraman S, and Haftka RT, Structural optimization complexity: what has Moore's law done for us?, Structural and Multidisciplinary Optimization, Vol. 28, No. 6, pp. 375-387, 2004.

[2] Sacks J, Welch WJ, Mitchell TJ, and Wynn HP, Design and Analysis of Computer Experiments, Statistical Science, Vol. 4 (4), pp. 409-435, 1989.

[3] Simpson TW, Peplinski JD, Koch PN, and Allen JK, Meta-models for computer based engineering design: Survey and recommendations. Engineering with Computers, Vol. 17, No. 2, pp. 129-150, 2001.

[4] Queipo NV, Haftka RT, Shyy W, Goel T, Vaidyanathan R, and Tucker, PK, Surrogate-based analysis and optimization, Progress in Aerospace Sciences, Vol. 41, pp. 1-28, 2005.

[5] Simpson TW, Toropov V, Balabanov V, and Viana FAC, Design and Analysis of Computer Experiments in Multidisciplinary Design Optimization: a Review of How Far We Have Come - or Not, in: Proceedings of the 12th AIAA/ISSMO Multidisciplinary Analysis and Optimization Conference, Victoria, Canada, September 10-12, 2008. AIAA 2008-5802.

[6] Kleijnen JPC, Design and analysis of simulation experiments, Springer Verlag, 2007.

[7] Forrester AIJ and Keane AJ, Recent advances in surrogate-based optimization, Progress in Aerospace Sciences, Vol. 45, No. 1-3, pp. 50-79, 2009.

[8] Montgomery DC, Design and analysis of experiments, John Wiley \& Sons, 2004.

[9] Simpson TW, Lin DKJ, and Chen W, Sampling Strategies for Computer Experiments: Design and Analysis, International Journal of Reliability and Applications, Vol. 2, No. 3, pp.209-240, 2001.

[10] Viana FAC, Haftka RT, and Steffen V., Multiple surrogates: how cross-validation errors can help us to obtain the best predictor, Structural and Multidisciplinary Optimization, available online (DOI: 10.1007/s00158-008-0338-0). 
[11] Viana FAC and Haftka RT, Using Multiple Surrogates for Metamodeling, 7th ASMO-UK/ISSMO International Conference on Engineering Design Optimization, Bath, UK, July 7-8, 2008.

[12] Samad A, Kim K, Goel T, Haftka RT, and Shyy W, Multiple surrogate modeling for axial compressor blade shape optimization, Journal of Propulsion and Power, Vol. 24, N. 2, pp. 302-310, 2008.

[13] Giunta AA, Wojtkiewicz SF, Eldred MS, Overview of modern design of experiments methods for computational simulations. 41 st AIAA Aerospace Sciences Meeting and Exhibit, Reno, NV, AIAA-2003-0649, 6-9 January 2003.

[14] Goel T, Haftka RT, Shyy W, and Watson LT, Pitfalls of using a single criterion for selecting experimental designs, International Journal for Numerical Methods in Engineering, Vol. 75, No. 2, pp. 127-155, 2008.

[15] Myers RH and Montgomery DC, Response Surface Methodology. Process and Product Optimization using Designed Experiments, John Wiley and Sons, 1995.

[16] McKay MD, Beckman RJ, and Conover WJ, A Comparison of Three Methods for Selecting Values of Input Variables from a Computer Code, Technometrics, Vol. 21, pp. 239-245, 1979.

[17] Iman RL, and Conover WJ, Small sample sensitivity analysis techniques for computer models, with an application to risk assessment, Communications in Statistics, Part A. Theory and Methods, Vol. 17, pp. 1749-1842, 1980.

[18] Kleijnen JPC, Sanchez SM, Lucas TW, and Cioppa TM, A user's guide to the brave new world of designing simulation experiments, INFORMS Journal on Computing, Vol. 17, No. 3, pp. 263-289, 2005.

[19] Audze P and Eglājs V, New Approach for Planning out of Experiments, Problems of Dynamics and Strengths, Vol. 35, 1977, pp. 104-107, Riga, Zinatne Publishing House (in Russian).

[20] Park JS, Optimal Latin-hypercube designs for computer experiments. Journal of Statistical Planning and Inference, Vol. 39, pp. 95-111, 1994.

[21] Morris MD, and Mitchell TJ, Exploratory designs for computational experiments, Journal of Statistical Planning and Inference, Vol. 43, pp. 381-402, 1995.

[22] Ye KQ, Li W, and Sudjianto A, Algorithmic construction of optimal symmetric latin hypercube designs. Journal of Statistical Planning and Inference, Vol. 90, pp. 145-159, 2000.

[23] Fang KT, Ma CX, and Winker P, Centered L2-discrepancy of random sampling and Latin hypercube design and construction of uniform designs, Mathematics of Computation, Vol. 71, pp. 275-296, 2002.

[24] Bates SJ, Sienz J and Toropov VV, Formulation of the optimal Latin hypercube design of experiments using a permutation genetic algorithm, 45th AIAA/ASME/ASCE/AHS/ASC Structures, Structural Dynamics and Materials Conference, AIAA2004-2011, Palm Springs, California, Apr. 19-22, 2004.

[25] Jin R, Chen W, and Sudjianto A, An Efficient Algorithm for Constructing Optimal Design of Computer Experiments, Journal of Statistical Planning and Inference, Vol. 134, pp. 268-287, 2005.

[26] Liefvendahl M and Stocki R, A study on algorithms for optimization of Latin hypercubes, Journal of Statistical Planning and Inference, Vol. 136, No. 9, pp. 3231-3247, 2006.

[27] van Dam E, Husslage B, den Hertog D, Melissen H, Maximin Latin hypercube designs in two dimensions, Operations Research, Vol. 55, No. 1, pp.158-169, 2007.

[28] Grosso A, Jamali A, and Locatelli M, Finding maximin Latin hypercube designs by Iterated Local Search heuristics, European Journal of Operational Research, Vol. 197, No.2, pp. 541-547, 2009.

[29] Viana FAC, SURROGATES ToolBox, http://fchegury.googlepages.com, 2009.

[30] Mathworks contributors, 2008, MATLAB ${ }^{\circledR}$ The language of technical computing, Version 7.6.0.324 R2008a, The MathWorks Inc.

[31] Cioppa TM and Lucas TW, Efficient nearly orthogonal and space-filling Latin hypercubes, Technometrics, Vol 49, No. 1, pp. 45-55, 2007.

[32] Hernandez AS, Breaking Barriers to Design Dimensions in Nearly Orthogonal Latin Hypercubes, PhD Thesis, Naval Postgraduate School, Monterey, CA, USA, 2008.

[33] Franco J, Carraro L, Roustant O, and Jourdan A, A radar-shaped statistic for testing and visualizing uniformity properties in computer experiments, Proceedings of the Joint ENBIS-DEINDE 2007 Conference: Computer Experiments versus Physical Experiments, Turin, Italy, April 11-13, 2007. 